\title{
miR-181d promotes pancreatic beta cell dysfunction by targeting IRS2 in gestational diabetes mellitus
}

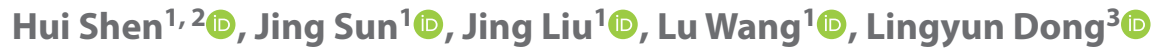 \\ ${ }^{1}$ Taikang Xianlin Drum Tower Hospital, Nanjing, China \\ ${ }^{2}$ The Affiliated Sir Run Run Hospital of Nanjing Medical University, Nanjing, China \\ ${ }^{3}$ Shanghai Public Health Clinical Center, Shanghai, China
}

\begin{abstract}
Objectives: Hyperglycemia that develops during pregnancy is a diagnostic criterion of gestational diabetes mellitus (GDM). Current studies have shown that the expression of miRNA-181d is significantly enhanced in the glomeruli of type 2 diabetic. However, the relationship between miR-181d and GDM has never been reported before.

Material and methods: The serum samples were collected from patients with GDM and subjected to qRT-PCR to verify the potential altered the miR-181d expression. In an in vitro GDM model, the miR-181d expression was induced by high glucose treatment, a miR-181d inhibitor was transfected into INS-1 cells to reduce miR-181d expression. Then, the level of insulin mRNA, cell viability, and content of total insulin were analyzed through ELISA, CCK-8 assay, and qRT-PCR assay. The relative apoptosis rates were detected by Annexin-V/PI assays. Finally, the shIRS2 transfection was performed to test whether in pancreatic $\beta$ cells, IRS2 had similar insulin-enhancing functions as the miR-181d inhibitor.

Results: MiR-181d expression level was positively correlated with fasting blood glucose levels and the inhibition of miR-181d reduced insulin resistance, enhanced cells viability and suppressed high-glucose-induced apoptosis. In addition, the suppression of miR-181d improved the functions of INS-1 cells by targeting IRS2.

Conclusions: In summary, this study indicated that miR-181d modulated the process of insulin signaling and cell viability and apoptosis in pancreatic $\beta$ cells by targeting IRS-2, suggesting that miR-181d inhibition is a potential target for GDM therapy. Key words: gestational diabetes mellitus; miR-181d; pancreatic beta cell; IRS2; insulin resistance
\end{abstract}

Ginekologia Polska 2021; 92, 8: 563-570

\section{INTRODUCTION}

Diabetes is linked to an abnormal metabolic condition that is characterized by insufficient blood glucose control or peripheral tissue resistance to insulin action [1, 2]. Hyperglycemia that develops during pregnancy is one of the symptoms of gestational diabetes mellitus (GDM) $[3,4]$. GDM, often beginning about 20-24 weeks of pregnancy, is defined as the glucose intolerance with onset or first recognition during pregnancy and whether the condition persists after pregnancy. GDM is currently the most general medical complication of pregnancy and affects approximately $3-8 \%$ of pregnancies [5, 6]. GDM drastically increases the various risks of a number of adverse events, such as respiratory distress syndrome, macrosomia, hyperbilirubinemia, hypoglycemia, metabolic syndrome, and type 2 diabetes, for fetuses and pregnant women [7-9]. However, the detailed molecular mechanism underlying the occurrence of GDM remains mostly unknown. The pathophysiology of GDM is related to defects in $\beta$-cell function $[10,11]$ and reductions in peripheral insulin sensitivity $[12,13]$. Therefore, finding the specific downstream factors of $\beta$ cells may help develop new therapeutic targets for GDM. MiRNAs are a class of small noncoding RNAs that regulate gene expression by repressing translation, degrading target mRNAs, and regulating the cell cycle and immune system homeostasis $[14,15]$. MiRNAs have been illustrated to be related with multiple sides of pancreatic development, glucose-stimulated insulin expression, and $\beta$-cell function or differentiation $[16,17]$. Recently, several studies have shown that circulating miRNAs in plasma or serum are putative early biomarkers of diabetes [18] and differentially expressed in GDM [19, 20]. The role of miRNAs in diabetes has been related with several

\author{
Corresponding author: \\ Hui Shen \\ Taikang Xianlin Drum Tower Hospital, No. 188 LingShan North Road, Qixia District, 210023 Nanjing, Jiangsu Province, China \\ e-mail:shenhui_1012@163.com
}

This article is available in open access under Creative Common Attribution-Non-Commercial-No Derivatives 4.0 International (CC BY-NC-ND 4.0) license, allowing to download articles and share them with others as long as they credit the authors and the publisher, but without permission to change them in any way or use them commercially. 
pathogenic characteristic, such as miR-410 and miR-200a stimulated the glucose level to regulate the insulin expression [21]. The overexpression of miR-30d and miR-29 also associated with insulin gene expression and insulin resistance [22]. Moreover, the expression of miRNA-181d is significantly enhanced in the glomeruli of type 2 diabetic mice and can reduce cellular triglycerides and cholesterol esters by inhibiting isocitrate dehydrogenase [23-25]. However, the relationship between miR-181d and GDM has never been reported before.

\section{Objectives}

We hypothesized that miR-181d can serve as a candidate biomarker for predicting GDM in early pregnancy. We concentrate on the expression level of miR-181d in GDM and consider its role in the functions of pancreatic $\beta$ cells. Then, we searched the signaling molecules and pathways that are regulated by miR-181d and identified the molecules that are the targets of miR-181d in regulating GDM.

\section{MATERIAL AND METHODS}

\section{Patients and blood samples}

The study was approved by the Ethics Committee of the Taikang Xianlin Drum Tower Hospital. The blood samples were obtained from normal pregnant women $(n=30)$ and pregnant women with $(n=25)$ GDM that were matched for age and body mass index (BMI) and requested to fasting blood glucose concentration measurements. All blood samples were stored at $-80^{\circ} \mathrm{C}$ until required.

\section{Cell culture}

Rat insulinoma (INS-1) cells were obtained from the cell bank of American type culture collection (ATCC) and cultured in RPMI 1640 medium (Gibco) with L-glutamine supplemented with $10 \mathrm{~mm}$ HEPES, $1 \mathrm{~mm}$ sodium pyruvate, $100 \mathrm{units} / \mathrm{mL}$ penicillin, $100 \mu \mathrm{g} / \mathrm{mL}$ streptomycin and $10 \%$ fetal bovine serum in a humidified atmosphere of $5 \% \mathrm{CO}_{2}$ and $37^{\circ} \mathrm{C}$. INS- 1 cells were subcultured when they achieved $90 \%$ confluence. Prior to experimental use, cells were precultured in serum free medium with different glucose condition (5.5, 11 or $22 \mathrm{~mm}$ ) overnight.

\section{Cell transfection}

INS- 1 cells were detached and plated at $2 \times 10^{5}$ cells per well into six well plates before transfection. When cell confluence reached $60-70 \%$, miR-181d inhibitor, NC inhibitor and short-hairpin IRS2 RNA (IRS2 shRNA) were transfected into cells using Lipofectamine 2000 and lentiviral packaging mix according to the reference instructions. After 48 hours, cells were collected for further experiments.

Quantitative real-time polymerase chain reaction (q-PCR)
Total RNA extracted from the cells and blood sample using TRIzol reagent (Thermo Fisher Scientific, USA) according to the reference instructions and reverse transcription of miRNA-181d to cDNA by PrimeScript RT reagent Kit (Takara, Japan). The specific primers for miRNA-181d were purchased from Applied Biosystems (TaqMan MicroRNA Assay, miRNA-181d, ID 001099). The gene expression level was quantitative by TaqMan MicroRNA assay kit on real-time PCR system (Applied Biosystems) were used according to the manufacturer's protocols. The mouse U6 was used as an internal control for normalized the miRNA-181d expression and comparisons calculated as the inverse log of $\triangle \Delta C T$ to give the relative fold change for all miRNA levels.

\section{Western Blot Analysis}

After different dose of glucose treatment and miR-181d inhibitor transfection, INS-1 cell were plural, were lysed with RIPA lysis buffer (Thermo Fisher Scientific, USA) and collected the total protein. The BCA kit was used to measure the protein concentration. $30 \mu \mathrm{g}$ proteins were separated by $10 \%$ sodium dodecyl sulfate-polyacrylamide (SDS) gel and transferred to Nitrocellulose (NC) membranes. After blocked in 5\% skim milk for two hours at normal atmospheric temperature, the membranes were then incubated with 1:2000 dilutions of the primary antibodies (IRS2 and $\beta$-actin, both antibodies purchase from Abcam, ab203051 and ab8227) at $4^{\circ} \mathrm{C}$ overnight. The membrane was washed and incubated with 1:5000 dilutions of the secondary antibodies for one hour at room temperature. Protein expression was determined by using enhanced chemiluminescence (ECL) detection System. Signal intensities were developed using Image Lab Software (version 3.0).

\section{Cell Apoptosis Assays}

The cells were harvested after treatment with different conditions and estimated the apoptosis cells by staining with annexin V/propidium iodide (PI) kit (Thermo Fisher Scientific, USA) according to the manufacturer's instructions. Finally, the fluorescence of cells was detected and analyzed by flow cytometry (Thermo Fisher Scientific, USA).

\section{CCK-8 assay}

The INS-1 cells viability were plural, were measured by Cell Counting Kit-8 (PA137267, Pierce) according to the manufacturer's instructions. The INS-1 cells were seeded at a density of $1 \times 10^{5}$ cells/well in a 96-well plate and treated with different concentration of glucose ( $5.5 \mathrm{~mm}$ to $22 \mathrm{~mm}$ ). After growth for different conditions, the cells were incubated by CCK-8 reagent for $20 \mathrm{~min}$. The absorbance value was assessed at $450 \mathrm{~nm}$ in a microplate reader. 


\section{Enzyme-linked immunosorbent assay (ELISA)}

The INS-1 cells were seeded and incubated for $24 \mathrm{~h}$. After treated with different concentration of glucose $(5.5 \mathrm{~mm}$ to $22 \mathrm{~mm}$ ) to stimulate release of insulin, the cells were lysed in lysis buffer, and then centrifuged for collected the supernatant. The supernatant was frozen at $-20^{\circ} \mathrm{C}$ until assayed. The secretion level of insulin from INS-1 cells were plural, were measured by insulin ELISA kit (EZHI-14K, Sigma-Aldrich) according to the manufacturer's instructions.

\section{Luciferase activity assay}

To investigate targets with significant change, the fragments of IRS2-3'-UTR with wide-type (IRS2 wt) and mutant (IRS2-mut) binding sites for miR-181d were inserted downstream of luciferase gene into pmirGLO luciferase Vectors (Promega, Madison, WI, USA). After the IRS2 wt and IRS2-mut, the cells were co-transfected with miR-181d inhibitor by Lipofectamine 2000. HEK293 cells were transfected with miR-181d inhibitor, along with mutant IRS2 reporter vector, the negative control (NC inhibitor) as needed within the experimental setup. After incubation, the luciferase reporter assay was used to applicated the cells, according to the manufacturer's instructions (Promega, Madison, WI, USA).

\section{Statistics}

All data were reported as mean $\pm \mathrm{SD}$. Analysis among multiple groups were carried out by ANOVA using the GraphPad Prism software 5.0. The connections between miR-181d with IRS2 and miR-181d with blood glucose were measured by Pearson correlation analysis. $P$ value $<0.05$ was considered statistically significant.

\section{RESULTS}

miR-181d is overexpress in gestational diabetes mellitus

Serum samples were collected from patients with GDM and nondiabetic pregnant women and subjected to qRT-PCR to verify the potential altered the miR-181d expression in the serum of patients with GDM. In the present study, the miR-181d level was markedly increased in patients with GDM compared with that in normal patients (Fig. 1A). The correlation between miR-181d and fasting blood glucose level was demonstrated via Pearson analysis. MiR-181d expression level was positively correlated with fasting blood glucose levels $(r=0.7182, p<0.001)$ (Fig. 1B). This result suggested that the overexpression of miR-181d might be a potential new diagnostic approach for GDM treatment. The INS-1 cell line presents many important characteristics, including high insulin content and response to glucose stimulation, of pancreatic $\beta$ cells. The INS- 1 cells were treated with different concentrations of glucose ranging from $5.5 \mathrm{~mm}$ to $22 \mathrm{~mm}$ for 4 days to elucidate the GDM-associated expression of miR-181d. As shown in Figure 1C, miR-181d was dose-dependently induced at glucose concentrations of $5.5 \mathrm{~mm}$ to $22 \mathrm{~mm}$. Taken together, these results indicated that miR-181d had a critical regulatory effect on GDM.

\section{Knockdown of miR-181d reduces insulin resistance in pancreatic $\beta$ cells}

Given that miR-181d expression was induced by high glucose treatment, a miR-181d inhibitor was transfected into INS-1 cells to reduce miR-181d expression (Fig. 2A). Then, level of insulin mRNA, cell viability, and insulin content were analyzed through ELISA, CCK-8 assay, and qRT-PCR assay. As depicted in Figure 2B, under high glucose stimulation (22 $\mathrm{mm}$ ), the viability of INS-1 cells in the miR-181d inhibition group significantly enhanced compared with that of cells in the blank and inhibitor NC groups. Compared with the blank and inhibitor NC groups, the insulin expression in response to glucose stimulation was significantly increased in the miR-181d inhibiting INS-1 cells. (Fig. 2C). However, cell viability and insulin secretion did not significantly differ among each group under low glucose conditions (5.5 mm).

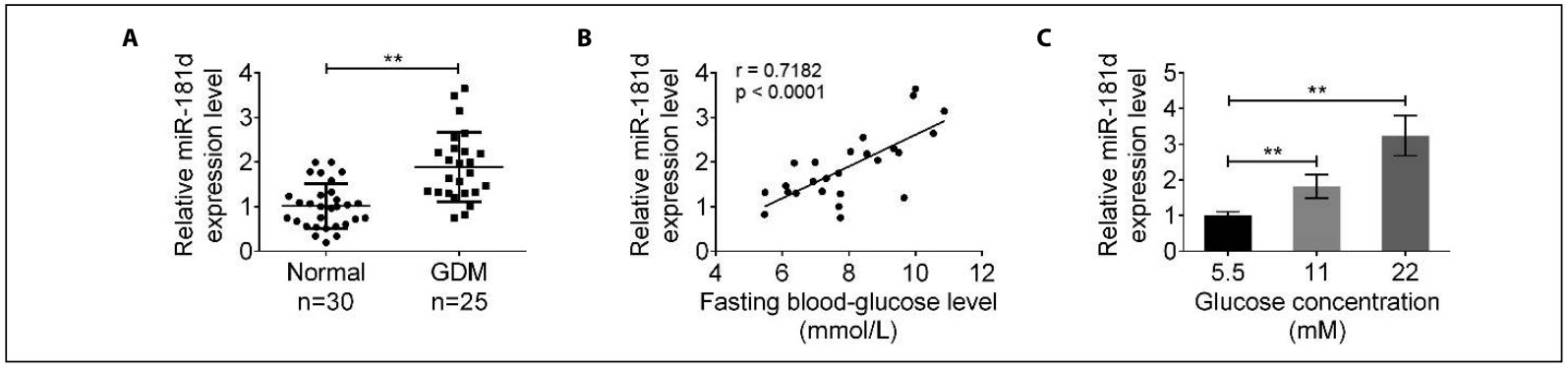

Figure 1. Up-regulation of miR-181d in GDM and its expression is positively correlated with blood glucose concentration; A. The expression levels of miR-181d were determined by qRT-PCR in blood samples from GDM pregnancies and healthy pregnancies. ${ }^{*} \mathrm{p}<0.01$ vs normal group; B. Correlation between miR-181d level and blood glucose determined by Pearson correlation analysis $(r=0.7182, p<0.0001)$; C. The expression levels of miR-181d were analyzed by qRT-PCR in INS-1 cells after treatment with different concentration of glucose. The values are means \pm SD. ** $\mathrm{p}<0.01$ vs $5.5 \mathrm{~mm}$ group 
A

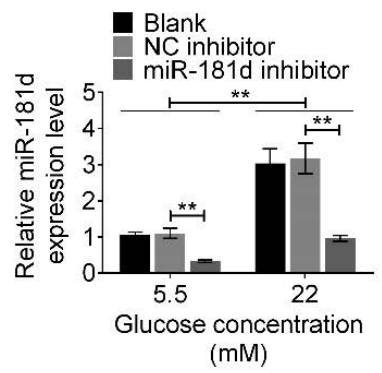

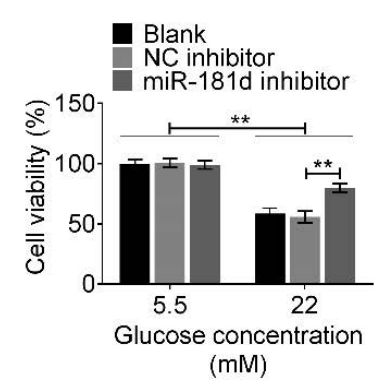

C

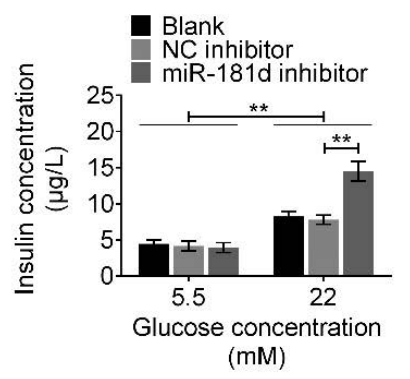

Figure 2. Knockdown of miR-181d enhanced the functions of pancreatic $\beta$ cell; $\mathbf{A}$. The transfection efficiency of miR-181d inhibitor in INS-1 cells after treatment with different concentration of glucose; B. Cell viability was determined by CCK-8 assay after transfection with miR-181d inhibitor in INS-1 cells; C. Total insulin secretion was measured by ELISA assay in INS-1 cells. The values are means \pm SD. ${ }^{* *} \mathrm{p}<0.01$ vs $5.5 \mathrm{~mm}$ group or NC inhibitor group

These results indicated that in INS-1 cells, the inhibition of miR-181d reduced insulin resistance and enhanced viability.

\section{Knockdown of miR-181d suppressed high- glucose-induced apoptosis in pancreatic $\beta$ cells}

Previous studies have demonstrated that high glucose concentration is toxic to cultured $\beta$ cells. The relative apoptosis rates, detected by Annexin-V/PI assays in INS-1 cells, were determined to validate whether miR-181d was indeed involved in the possible molecular mechanisms underlying the high-glucose-induced apoptosis of pancreatic $\beta$ cells. The data demonstrated that the group incubated with $22 \mathrm{~mm}$ glucose showed fewer live cells than the group treated with $5.5 \mathrm{~mm}$ glucose. Compared with that that of the high-glucose group, the relative rate of apoptosis in INS-1 cells was remarkably suppressed by the miR-181d inhibitor (Fig. 3). These results indicated that miR-181d affected high-glucose-induced apoptosis in INS-1 cells.

\section{MiR-181d functions as an IRS2 regulator}

Given that miRNAs function by regulating the expression of their target genes, bioinformatics analysis was performed to verify the potential targets of miR-181d. In accordance with the results of these analyses, we focused on the insulin receptor substrate IRS2, which plays a critical role in maintaining the adequate growth and survival of pancreatic $\beta$ cells. The WT IRS2 3'-UTR including the predicted miR-181d binding sites or the MT IRS2 3'-UTR without miR-181d binding sites were subcloned into a luciferase reporter vector (Fig. 4A) then subjected to a luciferase reporter assay. As shown in Figure 4A, cotransfection with the WT IRS2 3'-UTR vector and miR-181d inhibitor result in a remarkable increase in luciferase activity; however, the expression of luciferase in the 293T cells cotransfected with the MT IRS2 3'-UTR vector and miR-181d inhibitor showed no obvious change. Accordingly, IRS2 is a direct target gene of miR-181d. The expression levels of IRS2 were analyzed via Western blot analysis to identify whether miR-181d could modulate the expression of IRS2 in INS-1 cells. As depicted in Figure 4B, high glucose reduced IRS2 expression, whereas miR-181d inhibition reversed this effect.

\section{Suppression of miR-181d increaased the} functions of pancreatic $\beta$ cells by targeting IRS2

Considering that the inhibition of miR-181d led to a significant increase in the expression of IRS2, shIRS2 transfection was performed to test whether in pancreatic $\beta$ cells, IRS2 had similar insulin-enhancing functions as the miR-181d inhibitor. As illustrated in Figure 5A, miR-181d inhibition led to a significant increase in high-glucose-induced insulin expression and secretion. However, this effect was significantly reversed by shIRS2. CCK8 assay revealed that under high glucose conditions, cell viability in the shIRS2 treatment group reduced compared with that in the miR-181d inhibitor group (Figure 5B). Flow cytometry assay was conducted to further confirm whether shIRS2 expression could be negatively modulated by miR-181d under the condition of high-glucose-induced apoptosis. The cell apoptosis rate in the high-glucose treated group was promoted relative to that in the low-glucose group $(5.5 \mathrm{~mm})$ or high glucose $(22 \mathrm{~mm})+\mathrm{miR}-181 \mathrm{~d}$ inhibitor group. By contrast, after INS-1 cells were cotransfected with the miR-181d inhibitor and shIRS2, the reduction in cell apoptosis rate was reversed by shIRS2 treatment but not by the miR-181d inhibitor treatment. These results demonstrated that interference with IRS2 could change the regulatory effect of the miR-181d inhibitor on the pancreatic $\beta$ cells functions.

\section{DISCUSSION}

GDM results from a spectrum of physiological and genetic abnormalities and has severe negative effects on fetal and neonatal outcomes [26]. Pregnancy is gen- 


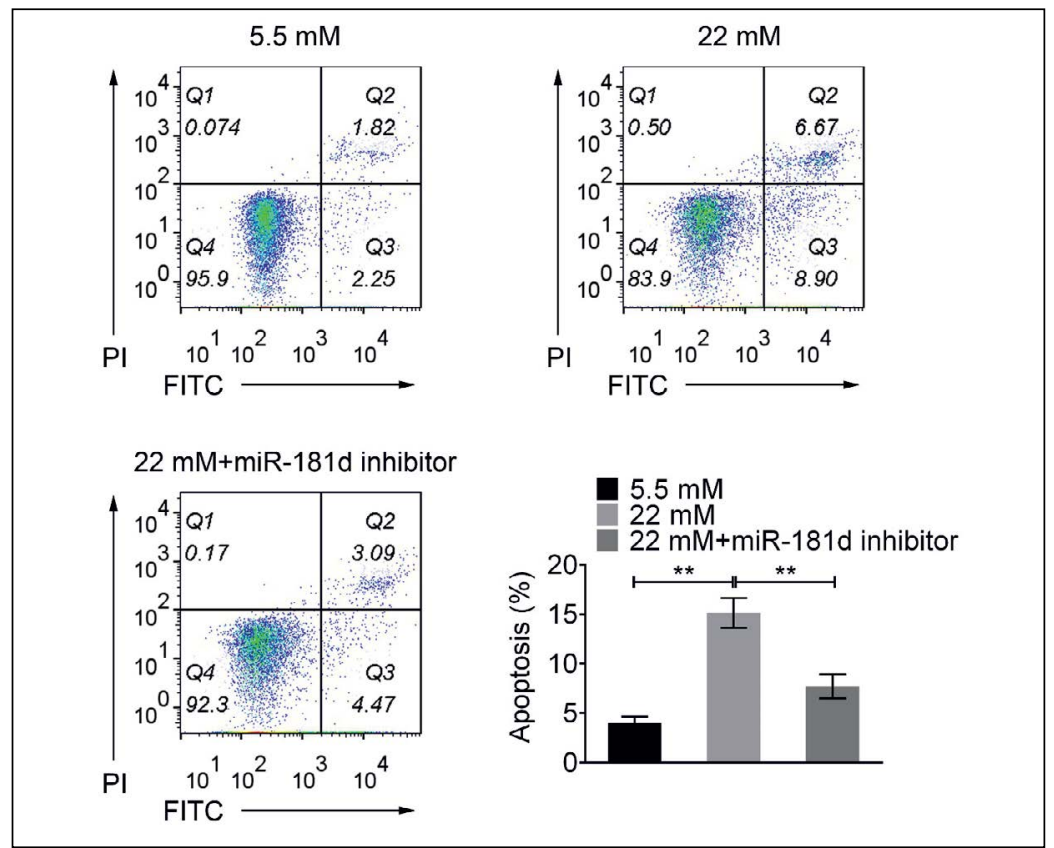

Figure 3. Knockdown of miR-181d suppresses cellular apoptosis of pancreatic $\beta$ cell. Cell apoptosis was analyzed by flow cytometry in INS-1 cells after transfection with the inhibitor and treatment with different concentration of glucose. The values are means \pm SD. ${ }^{* *} p<0.01$ vs 5.5 mm group or miR-181d inhibitor group

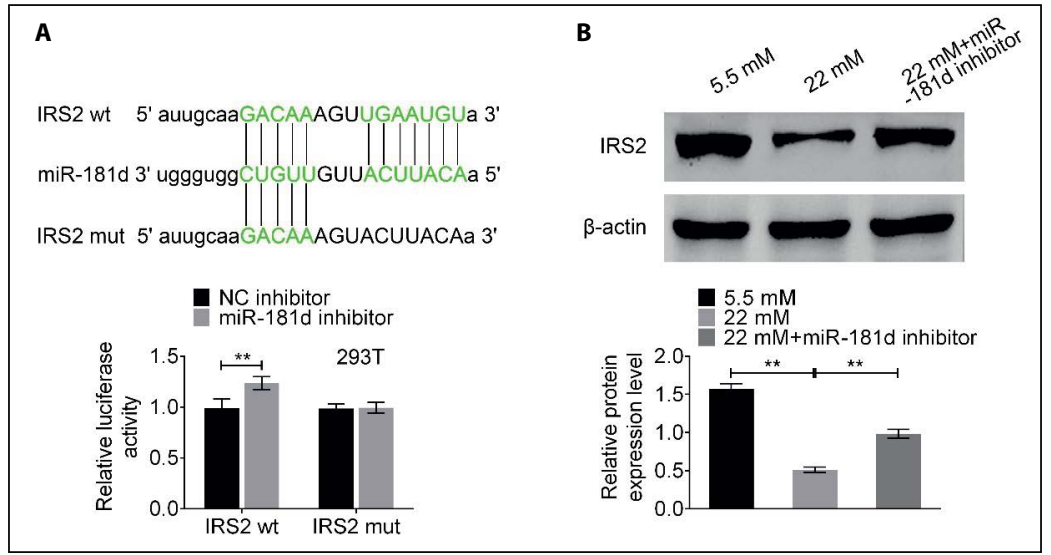

Figure 4. IRS2 was a direct target of miR-181d; A. IRS2 3'-UTR contains binding sites of miR-181d. (Up) Luciferase activity assay in HEK293T cells co-transfected with miR-181d inhibitor and luciferase reporters containing wild type or mutant type IRS2 (Down). ${ }^{* *} \mathrm{p}<0.01 \mathrm{vs}$ NC inhibitor group; B. IRS2 expression level in INS-1 cells was detected by western blot after transfection. The values are means \pm SD. ${ }^{* *} \mathrm{p}<0.01$ vs $5.5 \mathrm{~mm}$ group or miR-181d inhibitor group

erally associated with progressive insulin resistance that appears to result from the insulin-desensitizing effects of the hormonal products of the placenta $[27,28]$. Numerous studies have demonstrated that pancreatic $\beta$ cells increase their insulin secretion to compensate for insulin resistance during pregnancy, and the plasticity of $\beta$ cell function is a sign of normal glucose regulation during pregnancy [29, 30]. The serum miRNA signature that identifies GDM regulates the performance of the diversity of genes that are involved in the regulation of cell viability or apoptosis and glucose-stimulated insulin secretion and thus, may par- ticipate in diabetes $[19,31]$. Recently, miR-181d has been found to regulate the expression of $\mathrm{Bcl}-2$ family members and mitogen-activated protein kinase and is significantly up-regulated in the glomeruli of type 2 diabetic mice [32]. However, the accurate role of miR-181d in the rule of pancreatic $\beta$-cell proliferation, apoptosis, and insulin secretion remains unclear.

In the present study, we aimed to reveal essential roles of miR-181d in GDM. After comparing miR-181d expression in the blood samples of patients with diabetes that in the blood samples of normal subjects, we found that 


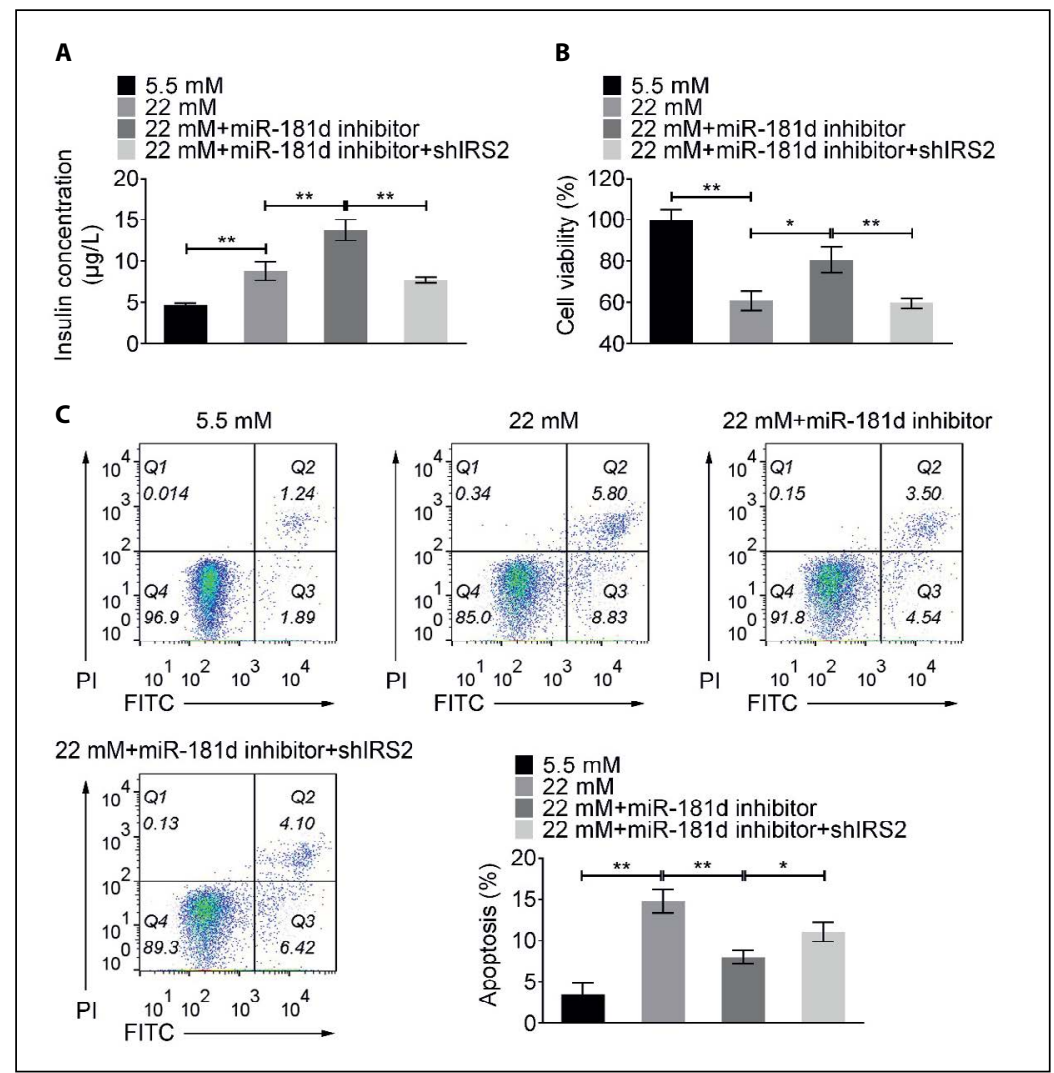

Figure 5. Knockdown of IRS2 reverses the regulatory effects of miR-181d inhibitor on the functions of pancreatic $\beta$ cells; $\mathbf{A}$. Total insulin secretion was measured by ELISA assay in INS-1 cells; B. Cell viability was determined by CCK- 8 assay after transfection in INS-1 cells; C. Cell apoptosis was analyzed by flow cytometry in INS-1 cells after transfection and treatment with different concentration of glucose. The values are means \pm SD. *, ** represent $5.5 \mathrm{~mm}$ or $22 \mathrm{~mm}+$ miR-181d inhibitor vs $22 \mathrm{~mm}$ or $22 \mathrm{~mm}+$ miR-181d inhibitor + shIRS2, p $<0.05, \mathrm{p}<0.01$

the expression of miR-181d was significantly increased in patients with diabetes. In patients with diabetes, plasma miR-181d expression level and blood glucose concentration have a significant positive correlation, indicating that the dysregulation of miR-181d is related to the progression of GDM. We transfected INS-1 cells with a miR-181d inhibitor to further investigate the mechanisms of miR-181d underlying the function of pancreatic $\beta$ cells in GDM. This study found that the suppression of miR-181d promoted cell viability and insulin expression while suppressing the cellular apoptosis of INS-1. These results were consistent with clinical data and further supported the concept that miR-181d plays a critical role in GDM.

At the post-transcriptional level, miRNAs regulate the expression of downstream target genes by binding to reverse complementary sequences. Our bioinformatics analysis results showed that IRS2 maintained the adequate growth and survival of pancreatic $\beta$ cells. IRS, a major cytoplasmic substrate in insulin signaling, modulates basic cellular functions and gene expression [33, 34]. IRS-2 is one of key members of the insulin receptor substrate family, which integrates the effects of insulin with those of IGF-1 to maintain insulin sensitivity and glucose homeostasis in $\beta$ cells [35]. The relative hyperinsulinemia could be compensated the peripheral insulin resistance caused by IRS2 disruption [36]. In Irs2 -/-, diabetes is promoted as $\beta$-cell mass decreases and insulin secretion fails [37]. In this study, when the miR-181d inhibitor was cotransfected with shIRS-2, luciferase activity was enhanced by the miR-181d inhibitor, suggesting that IRS-2 might be the target gene of miR-181d. In addition, the results of this research showed that the attenuation of IRS-2 with shRNA reversed the protective effects of miR-181d inhibition on insulin secretion and cell viability and the inhibitory effect on the apoptosis of INS-1 cells under high-glucose conditions. These results suggested that miR-181d inhibition exerted a functional role in GDM by targeting IRS- 2 .

\section{CONCLUSIONS}

In summary, this study indicated that miR-181d involved inthe insulin signaling and cell viability and apoptosis in pancreatic $\beta$ cells by targeting IRS- 2 , suggesting that miR-181d inhibition is a potential target for GDM therapy. 


\section{Acknowledgements}

Not applicable.

\section{Funding}

This work was supported by Science and Technology Development Fund of Nanjing Medical University (Grant No. 2017NJMUZD133).

\section{Conflict of interest}

The authors state that there are no conflicts of interest to disclose.

\section{Ethics approval}

Ethical approval was obtained from the Ethics Committee of the Taikang Xianlin Drum Tower Hospital.

\section{Statement of Informed Consent}

Written informed consent was obtained from a legally authorized representatives for anonymized patient information to be published in this article.

\section{Availability of data and materials}

All data generated or analyzed during this study are included in this published article.

\section{Authors' contributions}

HS and JS designed the study, supervised the data collection, JL and LW analyzed the data, interpreted the data, LD prepare the manuscript for publication and reviewed the draft of the manuscript. All authors have read and approved the manuscript.

\section{REFERENCES}

1. Chaudhury A, Duvoor C, Reddy Dendi VS, et al. Clinical review of antidiabetic drugs: implications for type 2 diabetes mellitus management. Front Endocrinol (Lausanne). 2017; 8: 6, doi: 10.3389/fendo.2017.00006, indexed in Pubmed: 28167928.

2. Czech $M$. Insulin action and resistance in obesity and type 2 diabetes. Nature Medicine. 2017; 23(7): 804-814, doi: 10.1038/nm.4350.

3. Plows JF, Stanley JL, Baker PN, et al. The pathophysiology of gestational diabetes mellitus. Int J Mol Sci. 2018; 19(11): 3342, doi: 10.3390/ijms19113342, indexed in Pubmed: 30373146.

4. Lowe WL, Scholtens DM, Kuang A, et al. HAPO Follow-up Study Cooperative Research Group. Hyperglycemia and adverse pregnancy outcome follow-up study (HAPO FUS): maternal gestational diabetes mellitus and childhood glucose metabolism. Diabetes Care. 2019;42(3):372-380, doi: 10.2337/dc18-1646, indexed in Pubmed: 30655380.

5. Mclntyre H, Catalano $P$, Zhang C, et al. Gestational diabetes mellitus. Nat Rev Dis Primers. 2019; 5(1):47, doi: 10.1038/s41572-019-0098-8, indexed in Pubmed: 31296866.

6. Wang $C$, Wei $Y$, Zhang $X$, et al. A randomized clinical trial of exercise during pregnancy to prevent gestational diabetes mellitus and improve pregnancy outcome in overweight and obese pregnant women. Am J Obstet Gynecol. 2017; 216(4):340-351, doi: 10.1016/j.ajog.2017.01.037, indexed in Pubmed: 28161306.

7. Kc K, Shakya S, Zhang H. Gestational diabetes mellitus and macrosomia: a literature review. Ann Nutr Metab. 2015; 66(Suppl 2): 14-20, doi: 10.1159/000371628, indexed in Pubmed: 26045324.
8. Landon MB, Catalano PM, Gabbe SG. Diabetes mellitus complicating pregnancy. In: Landon MB, Galan HL, Jauniaux ERM. ed. Gabbe's obstetrics essentials: normal and problem pregnancies. , Elsevier 2018: 674-691.

9. Hartling L, Dryden DM, Guthrie A, et al. Benefits and harms of treating gestational diabetes mellitus: a systematic review and meta-analysis for the U.S. Preventive Services Task Force and the National Institutes of Health Office of Medical Applications of Research. Ann Intern Med. 2013; 159(2): 123-129, doi: 10.7326/0003-4819-159-2-201307160-00661, indexed in Pubmed: 23712381.

10. Su Jb, Wang Xq, Chen Jf, et al. Glycemic variability in gestational diabetes mellitus and its association with $\beta$ cell function. Endocrine. 2013; 43(2): 370-375, doi: 10.1007/s12020-012-9753-5, indexed in Pubmed: 22815046.

11. Kelstrup L, Damm P, Mathiesen ER, et al. Insulin resistance and impaired pancreatic $\beta$-cell function in adult offspring of women with diabetes in pregnancy. J Clin Endocrinol Metab. 2013; 98(9): 3793-3801, doi: 10.1210/jc.2013-1536, indexed in Pubmed: 23796568.

12. Catalano PM. Trying to understand gestational diabetes. Diabet Med. 2014; 31(3): 273-281, doi: 10.1111/dme.12381, indexed in Pubmed: 24341419.

13. Xiang $\mathrm{AH}$, Takayanagi $\mathrm{M}, \mathrm{Black} \mathrm{MH}$, et al. Longitudinal changes in insulin sensitivity and beta cell function between women with and without a history of gestational diabetes mellitus. Diabetologia. 2013; 56(12): 2753-2760, doi: 10.1007/s00125-013-3048-0, indexed in Pubmed: 24030069.

14. Wilczynska A, Bushell M. The complexity of miRNA-mediated repression Cell Death Differ. 2015; 22(1):22-33, doi: 10.1038/cdd.2014.112, indexed in Pubmed: 25190144.

15. Gurtan AM, Sharp PA. The role of miRNAs in regulating gene expression networks. J Mol Biol. 2013; 425(19): 3582-3600, doi: 10.1016/j. jmb.2013.03.007, indexed in Pubmed: 23500488.

16. Cote GA, Gore AJ, McElyea SD, et al. A pilot study to develop a diagnostic test for pancreatic ductal adenocarcinoma based on differential expression of select miRNA in plasma and bile. Am J Gastroenterol. 2014; 109(12): 1942-1952, doi: 10.1038/ajg.2014.331, indexed in Pubmed: 25350767.

17. Lahmy R, Soleimani M, Sanati MH, et al. MiRNA-375 promotes beta pancreatic differentiation in human induced pluripotent stem (hiPS) cells. Mol Biol Rep. 2014; 41(4): 2055-2066, doi: 10.1007/s11033-0143054-4, indexed in Pubmed: 24469711.

18. Chakraborty C, Doss CG, Bandyopadhyay S, et al. Influence of miRNA in insulin signaling pathway and insulin resistance: micro-molecules with a major role in type-2 diabetes. Wiley Interdiscip Rev RNA. 2014 5(5): 697-712, doi: 10.1002/wrna.1240, indexed in Pubmed: 24944010.

19. Collares CVA, Evangelista AF, Xavier DJ, et al. Identifying common and specific microRNAs expressed in peripheral blood mononuclear cell of type 1 , type 2, and gestational diabetes mellitus patients. BMC Res Notes. 2013; 6: 491, doi: 10.1186/1756-0500-6-491, indexed in Pubmed: 24279768.

20. Zhu Y,Tian F, Li H, et al. Profiling maternal plasma microRNA expression in early pregnancy to predict gestational diabetes mellitus. Int J Gynaecol Obstet. 2015; 130(1): 49-53, doi: 10.1016/j.ijgo.2015.01.010, indexed in Pubmed: 25887942.

21. Hennessy $E$, Clynes $M$, Jeppesen PB, et al. Identification of microRNAs with a role in glucose stimulated insulin secretion by expression profiling of MIN6 cells. Biochem Biophys Res Commun. 2010; 396(2): 457-462, doi: 10.1016/j.bbrc.2010.04.116, indexed in Pubmed: 20417623.

22. He A, Zhu L, Gupta N, et al. Overexpression of micro ribonucleic acid 29 , highly up-regulated in diabetic rats, leads to insulin resistance in 3T3-L1 adipocytes. Mol Endocrinol. 2007; 21(11): 2785-2794, doi: 10.1210/me.2007-0167, indexed in Pubmed: 17652184.

23. Zhu X, Zhang C, Fan Q, et al. Inhibiting microrna-503 and microrna-181d with losartan ameliorates diabetic nephropathy in KKAy mice. Med Sci Monit. 2016; 22: 3902-3909, doi: 10.12659/msm.900938, indexed in Pubmed: 27770539 .

24. Dewanjee $S$, Bhattacharjee N. MicroRNA: a new generation therapeutic target in diabetic nephropathy. Biochem Pharmacol. 2018; 155: 32-47, doi: 10.1016/j.bcp.2018.06.017, indexed in Pubmed: 29940170.

25. Yap CS, Sinha RA, Ota $S$, et al. Thyroid hormone negatively regulates CDX2 and SOAT2 mRNA expression via induction of miRNA-181d in hepatic cells. Biochem Biophys Res Commun. 2013;440(4):635-639, doi: 10.1016/j.bbrc.2013.09.116, indexed in Pubmed: 24103759.

26. Buchanan T, Xiang A, Page K. Gestational diabetes mellitus: risks and management during and after pregnancy. Nat Rev Endocrinol. 2012; 
8(11): 639-649, doi: 10.1038/nrendo.2012.96, indexed in Pubmed: 22751341.

27. Yogev Y, Ben-Haroush A, Hod M. Pathogenesis of gestational diabetes mellitus. In: Hod M, Jovanovic LG, Di Renzo GC, De Leiva A, Langer O. ed. Textbook of Diabetes and Pregnancy. CRC Press, London 2008: 46.

28. Svensson $\mathrm{H}$, Wetterling $\mathrm{L}$, Bosaeus $\mathrm{M}$, et al. Body fat mass and the proportion of very large adipocytes in pregnant women are associated with gestational insulin resistance. Int J Obes (Lond). 2016; 40(4): 646-653, doi: 10.1038/ijo.2015.232, indexed in Pubmed: 26563815.

29. Ohara-Imaizumi M, Kim H, Yoshida $M$, et al. Serotonin regulates glucose-stimulated insulin secretion from pancreatic $\beta$ cells during pregnancy. Proc Natl Acad Sci U S A. 2013; 110(48): 19420-19425, doi: 10.1073/pnas.1310953110, indexed in Pubmed: 24218571.

30. Fryirs MA, Barter PJ, Appavoo M, et al. Effects of high-density lipoproteins on pancreatic beta-cell insulin secretion. Arterioscler Thromb Vasc Biol. 2010; 30(8): 1642-1648, doi: 10.1161/ATVBAHA.110.207373, indexed in Pubmed: 20466975.

31. Mao $\mathrm{Y}$, Mohan R, Zhang $\mathrm{S}$, et al. MicroRNAs as pharmacological targets in diabetes. Pharmacol Res. 2013; 75: 37-47, doi: 10.1016/j. phrs.2013.06.005, indexed in Pubmed: 23810798.

32. Ouyang YB, Lu Y, Yue S, et al. miR-181 targets multiple Bcl-2 family members and influences apoptosis and mitochondrial function in astrocytes. Mitochondrion. 2012; 12(2): 213-219, doi: 10.1016/j. mito.2011.09.001, indexed in Pubmed: 21958558.

33. Bomfim TR, Forny-Germano L, Sathler LB, et al. An anti-diabetes agent protects the mouse brain from defective insulin signaling caused by Alzheimer's disease- associated $A \beta$ oligomers. J Clin Invest. 2012; 122(4): 1339-1353, doi: 10.1172/JCI57256, indexed in Pubmed: 22476196.

34. Alharbi KK, Khan IA, Munshi A, et al. Association of the genetic variants of insulin receptor substrate 1 (IRS-1) with type 2 diabetes mellitus in a Saudi population. Endocrine. 2014; 47(2): 472-477, doi: 10.1007/s12020014-0177-2, indexed in Pubmed: 24493031.

35. Guo S. Molecular basis of insulin resistance: the role of IRS and foxo1 in the control of diabetes mellitus and its complications. Drug Discov Today Dis Mech. 2013; 10(1-2): e27-e33, doi: 10.1016/j.ddmec.2013.06.003, indexed in Pubmed: 24015152.

36. Guo S. Insulin signaling, resistance, and the metabolic syndrome: insights from mouse models into disease mechanisms. J Endocrinol. 2014; 220(2): T1-T23, doi: 10.1530/JOE-13-0327, indexed in Pubmed: 24281010.

37. Tiano JP, Mauvais-Jarvis F. Importance of oestrogen receptors to preserve functional $\beta$-cell mass in diabetes. Nat Rev Endocrinol. 2012; 8(6): 342-351, doi: 10.1038/nrendo.2011.242, indexed in Pubmed: 22330739 . 